\title{
Heat Conduction: Hyperbolic Self-similar Shock-waves in Solid Medium
}

\section{Barna IF $^{1 *}$ and Kersner $\mathbf{R}^{2}$}

${ }^{1}$ Wigner Research Center of the Hungarian Academy of Sciences, Konkoly Thege Miklós út 29-33, Budapest 1121, Hungary

${ }^{2}$ Department of Mathematics and Informatics, University of Pécs, PMMK, Boszorkány u. 2, Pécs, Hungary

\begin{abstract}
Analytic solutions for cylindrical thermal waves in solid medium are given based on the nonlinear hyperbolic system of heat flux relaxation and energy conservation equations. The Fourier-Cattaneo phenomenological law is generalized where the relaxation time and heat propagation coefficient have a general power law temperature dependence. From such laws one cannot form a second order parabolic or telegraph-type equation. We consider the original non-linear hyperbolic system itself with the self-similar Ansatz for the temperature distribution and for the heat flux. As results continuous.
\end{abstract}

Keywords: Self-similar solution; Non-linear heat conduction; Shock wave; Cattaneo heat conduction law

\section{Introduction}

Analytic solutions for cylindrical thermal waves in solid medium are given based on the nonlinear hyperbolic system of heat flux relaxation and energy conservation equations. The Fourier-Cattaneo phenomenological law is generalized where the relaxation time and heat propagation coefficient have a general power law temperature dependence. From such laws one cannot form a second order parabolic or telegraph-type equation. We consider the original non-linear hyperbolic system itself with the self-similar Ansatz for the temperature distribution and for the heat flux. As results continuous and shockwave solutions are presented. For physical establishment numerous materials with various temperature dependent heat conduction coefficients are mentioned.

44.90.+c, 02.30.Jr

In contemporary heat transport theory (ever since Maxwell's paper [1]) it is widely accepted in the literature that only for stationary and weakly non-stationary temperature fields the constitutive equation assumes that a temperature gradient $\nabla T$ instantaneously produces heat flux $q$ according to the Fourier law.

$$
q(x, t)=-\kappa \nabla T(x, t)
$$

Combining this equation with the energy conservation law the usual parabolic heat conduction equation is given. Heat conduction mechanisms can be classified via the temperature dependence of the coefficient $\kappa: T^{v}$. There are three different cases of thermal conductivity, normal heat conduction which obeys the Fourier law $(v=0)$, slow $(v>0)$ and fast heat conduction $-2<v<0$.

In plasma physics if the temperature range is between $10^{5} \mathrm{~K}$ and $10^{8} \mathrm{~K}$ then the coefficient of the heat conductivity $\kappa$ depends on the temperature and density of the material. It is usually assumed to have a power dependence $\kappa=\kappa_{0} T^{v} v^{\mu}$ where $v=1 / \rho$ is the specific volume the coefficient $\kappa_{0}$ and the exponents $v, \mu$ depend on the heat conduction mechanism [2]. With radiation heat conduction one has $4 \leq v \leq 6,2 m m 1 \leq \mu \leq 2$; with electron heat conduction and fully ionized plasma $v=5 / 2,2 m m \mu=0$. For magnetically confined nonneutral plasma the classical heat conduction coefficient is the following [3] $\kappa \approx \frac{c_{1}}{\sqrt{T}} \ln \left[c_{2} T^{3 / 2}\right]$. Parabolic thermal wave theory is based on this approach $[2,4]$. In plasmas heat conduction is strongly coupled to flow properties which we will not consider in the following. The linear parabolic theory predicts infinite speed of propagation which is known as the "paradox of heat conduction" (PHC). The following two theories resolve this contradiction.

However, if the time scale of local temperature variation is very small, Eq. (1) is replaced by

$$
q(x, t=\tau)=-\kappa \nabla T(x, t)
$$

Where $\tau$ is called the thermal relaxation time. This is a thermodynamic property of the materials which was determined experimentally for large number of materials. Although $\tau$ turns out to be very small in many instances e.g. is of order of picoseconds for most metals, there are several materials where this is not the case, most notably sand (21 s), $\mathrm{H}$ acid (25 s), $\mathrm{NaHCO}_{3}(29 \mathrm{~s})$, and biological tissue (1-100 s) [5].

Unlike the Fourier's heat conduction law, this constitutive equation is non-local in time. The desired local character can be restored with the Taylor expansion of $q$ by time which is usually truncated at the first order namely

$$
q(x, t)+\tau \frac{\partial q(x, t)}{\partial t}=-\kappa \nabla T(x, t) .
$$

This is the well-known Cattaneo heat conduction law [6] the second term on the left hand side is known as the "thermal intertia". Combining this constitutive equation with the energy conservation yields the hyperbolic telegraph heat conduction equation where $\tau$ and are constants. Hyperbolic equations usually ensure finite propagation velocity. Unfortunately, the usual telegraph equations has no selfsimilar solutions which would be a desirable physical property. In the work of [7] a non-autonomous telegraph-type heat conduction equation is presented with self-similar non-oscillating compactly supported solutions. A review with a large number of physical models of heat waves can be found in $[5,8]$. A recent work on the speed of heat waves was published by [9].

${ }^{*}$ Corresponding author: Barna IF, Wigner Research Center of the Hungarian Academy of Sciences, Konkoly Thege Miklós út 29-33, Budapest, Hungary, Tel: +36-1-392-2222/3504; Fax: +36-1-392-2598; E-mail: barna.imre@wigner.mta.hu

Received February 29, 2016; Accepted April 14, 2016; Published May 03, 2016

Citation: Barna IF, Kersner R (2016) Heat Conduction: Hyperbolic Selfsimilar Shock-waves in Solid Medium. J Generalized Lie Theory Appl S2: 010. doi:10.4172/1736-4337.S2-010

Copyright: (c) 2016 Barna IF, et al. This is an open-access article distributed unde the terms of the Creative Commons Attribution License, which permits unrestricted use, distribution, and reproduction in any medium, provided the original author and source are credited. 
Our starting point is the following

$$
\begin{aligned}
& q_{t}=-\frac{q}{\tau}-\frac{\kappa}{\tau} T_{r}, \\
& c_{0} T_{t}=-q_{r}-\frac{q}{r} .
\end{aligned}
$$

The first equation of the system is the generalized Fourier-Cattaneo heat conduction law and the second one is the energy conservation condition for the radial coordinate. The heat flux $q=q(r, t)$ and the temperature $T=T(r, t)$ have radial coordinate and time dependence. The subscripts $\mathrm{r}$ and $\mathrm{t}$ denote the partial derivatives with respect to the radial coordinate and the time, respectively. (From now on we investigate the radial coordinate of a cylindrical symmetric problem as spatial dependence.) The parameter $c_{0}=\rho c$ where $\rho$ is the mass density and $c$ is the specific heat. Second order effects such as compressibility are neglected ( $\rho$ and $c$ are constants during the process).

In the following we shall suppose that the heat conduction coefficient and the thermal relaxation depend on temperature on the following way:

$$
\kappa=\kappa_{0} T^{\omega}, 1 c m \tau=\tau T^{-\delta}
$$

The $\kappa_{0}$ and $\tau_{0}$ are real numbers with the proper physical dimensions. Now our dimensionless system reads:

$$
\begin{gathered}
q_{t}=-T^{\varepsilon} q-T^{\varepsilon+\omega} T_{r} \\
T_{t}=-q_{r}-\frac{q}{r} .
\end{gathered}
$$

There are various phenomenological heat conduction laws available for all kind of solids, without completeness we mention some wellknown examples. For pure metals according to [10] (Page 275 Eq. 27.3) the Wiedemann-Franz law the thermal conductivity is proportional with the electrical conductivity times the temperature $\kappa=\sigma L T$ The proportionality constant $L$ is the so called Lorentz number with the approximate numerical value of $2.44 \times 10^{-8} \mathrm{~W} \Omega \mathrm{K}^{-2}$. For exact numerical data for various metals see [11]. The relaxation time $\tau$ is proportional to the heat conduction coefficient divided by the temperature. For metals with impurities the thermal resistivity (inverse of the thermal conductivity) is $\kappa^{-1}=A T^{2}+B T^{-1}$ where $\mathrm{A}$ and $\mathrm{B}$ can be obtained from microscopic calculation based on quantum mechanics [10] (Page 297 Eq. 40.11)

A hard-sphere model for dense fluids from [12] derives a relation where the heat flux $q(x, t)=a \nabla T(x, t)+q^{2}(x, t)$ which certainly means a nonlinear heat propagation process. For the heat conduction in nanofluid suspensions [13] derives the $\kappa \approx c /\left(T_{2}-T_{1}\right)$ law with additional time dependence. Another exotic and very promising new materials are the carbon nanotubes which have exotic heat conduction properties. Small et al. [14] performed heat conductivity measurements and found that at low temperatures there are two distinct regimes $\kappa(T): T^{2.5}(T<50 K)$ and $\kappa(t): T^{2}(50<T<150 K)$. Beyond this regime there is a deviance from this quadratic temperature dependence and the maximum $\kappa$ value lies at $320 \mathrm{~K}$. Above this value - at large temperatures - there is a $\kappa(T)$ : $1 / T$ dependence according to [15]. Additional nanoscale systems (like silicon films, or multiwall carbon nanotubes) have exotic temperature dependent heat conduction coefficients as well, for more see [16]. For encased graphene the heat conduction coefficient is $\kappa: T^{\beta}$ where $1.5<\beta<2$ at low temperature $(T<150 K)$ [17]. A recent review of thermal properties of graphene and nanostructured carbon materials can be found in [18]

Our model is presented to describe the heat conduction of any kind of solid state without additional restrictions, therefore room or even higher temperature can be considered with large negative $\omega$ exponents.

Even from these examples we can see that it has a need to investigate the general heat conduction problem, where the coefficients have general power law dependence. form.

We look for the solutions of $(7,8)$ in the most general self-similar

$$
T=t^{\alpha} f(\eta), q=t^{-\delta} g(\eta)
$$

For a better transparency in the following we introduce a new variable $\eta=\frac{r}{t^{\beta}}$, where $\alpha, \beta, \delta$ are all real numbers.

The similarity exponents $\alpha, \delta$ and $\beta$ are of primary physical importance since $\alpha, \delta$ represents the rate of decay of the magnitude $T$ or $q$, while $\beta$ is the rate of spread (or contraction if $<0$ ) of the space distribution as time goes on. Self-similar solutions exclude the existence of any single time scale in the investigated system.

We substitute (9) into (7) and (8). It can be checked that

$\alpha=\frac{1}{\omega+1}, \quad 3 m m \beta=\frac{1}{2(\omega+1)}, \quad 3 m m \delta=\frac{2 \quad 3}{2(\omega+1)}, \quad 3 m m \varepsilon=\omega \quad 1$.

Then we can obtain the shape functions $\mathrm{f}$ and $\mathrm{g}$ the following ordinary differential equation (ODE) system

$$
\begin{aligned}
& \delta g+\beta \eta g^{\prime}=g f^{\omega+1}+f^{2 \omega+1} f^{\prime}, \\
& (\eta g)^{\prime}=\beta\left(\eta^{2} f\right)^{\prime}
\end{aligned}
$$

where prime means derivation with respect to $\eta$.

The first lucky moment is that (12) relates $\mathrm{f}$ and $\mathrm{g}$ in a simple way $\mathrm{g}=\beta \eta f(13)$

if the $\alpha=2 \beta$ universality relation is fulfilled.

Note, that we can immediately read how the self-similar solutions of the temperature distribution $T$ and the heat flux $q$ depend on $\omega$

$$
T=t^{\frac{-1}{\omega+1}} f\left(\frac{r}{\frac{1}{t^{2(\omega+1)}}}\right), \quad 8 m m q=t^{\frac{2 \omega+3}{2(\omega+1)}} g\left(\frac{r}{\frac{1}{t^{2(\omega+1)}}}\right) .
$$

The parameter dependence of the complete heat conduction coefficient and relaxation time can be expressed via $\omega$ as well

$$
\kappa=\kappa_{0} t^{\frac{-\omega}{\omega+1}} f^{\omega}\left(\frac{r}{t^{\frac{1}{2(\omega+1)}}}\right), \quad \tau=\kappa_{0} t^{-1} f^{\omega+1}\left(\frac{r}{t^{\frac{1}{2(\omega+1)}}}\right) .
$$

Recall that $>-1$. These are already very informative and useful relations to investigate the global properties of the solutions, note that such kind of analysis are available for large number of complex mechanical and flow problems [19].

Substituting these relations back to Eq. (11) after some algebra we arrive at the following non-linear first-order ODE

$$
\frac{d f}{d \eta^{2}}\left(\beta^{2} \eta^{2}-f^{2 \omega+1}\right)=\frac{\beta f}{2}\left[f^{\omega+1}-(2 \beta+1)\right] .
$$

Put $y=\eta^{2}$ and $x=f$. With this notation eq. (16) becomes linear for $y(x)$ (this is the second lucky moment of investigation):

$$
\frac{d y}{d x}=\frac{y(x)-4(\omega+1)^{2} x^{2 \omega+1}}{x\left[(\omega+1) x^{\omega+1}-\omega-2\right]} .
$$

Plainly, $f x$ is a solution to eq. (16). If $y(x)$ the solution of eq. (17) is strictly monotonic then so is the inverse function $f=x$ and no discontinuity. However if $y(x)$ is not monotonic on some interval $\left(x_{1}\right.$, 
$\left.x_{2}\right)$ and has a turning point at $x_{0} \varepsilon\left(x_{1}, x_{2}\right)$ then the inverse $(f=x)$ has sense on $[0, y(x)]$ only. One sets $f=0$ for $y>y\left(x_{0}\right)$ and the discontinuity a $y\left(x_{0}\right)$ is apparent. The analytical investigation of the linear equation (17) is, in general easier than of eq (16). In some cases (for some $\omega \mathrm{s}$ ) one can have more explicit or almost explicit solutions.

\section{There are two examples:}

The first case is for $\omega=0,(\alpha=1, \beta=1 / 2, \delta=3 / 2, \varepsilon=1)$.

This example was studied by [20] in some details. The corresponding ODE (17) reads $y^{\prime}=(y-4 x) / x(x-2)$ which has a solution $y=8+[(x-2) / x]^{1 / 2}\left[c_{1}-8 \ln (\sqrt{x}+\sqrt{x-2})\right]$ where $c_{1}$ is a constant.

It is clear that must be $\mathrm{x} \geq 2$ and $y(x)$ is monotonic for $x>2$ until $x_{0}$ where $y=0$. This means that $x(y)$ exists and monotonic on some interval $\left[0, y_{0}\right], x\left(y_{0}\right)=2$; for $y \geq y_{0}$ we have $x(y)=0$ so the discontinuity. For a better understanding Figure 1a presents the graph of solution of Eq. (17) through the point $(3,0.5)$. The inverse of this function for $x>2$ is shown on Figure $1 \mathrm{~b}$ (the nonzero part). The solid line is a solution through the $f(0)=10.8$ point. Figure 2 presents the theoretical shockwave propagation of the temperature distribution $T(r, t)$ for $\omega=0$.

The second case is for $\omega=-1 / 2,(\alpha=2, \beta=1, \delta=2, \varepsilon=1 / 2)$.

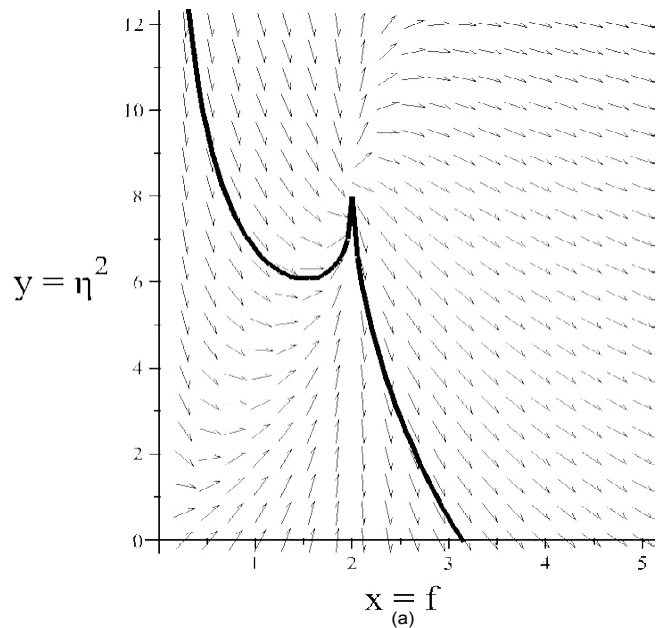

Now Eq. (17) takes the form of $\frac{d y}{d x}=2(y-1) /[x(\sqrt{x}-3)]$. It can be checked that $y=c_{2} x^{-2 / 3}\left(x^{1 / 2}-3\right)^{4 / 3}$ is a solution for any $c_{2}>0$. Take $c_{2=0}$. The function $y(x)$ is monotonic on $(0,9), y(9)=0$. Returning to original variables we have $f=9 /\left[\left(\eta^{2}\right)^{3 / 4}+1\right]^{2}$ (which is plainly less than 9!) According to eq. (14) temperature and heat flux distributions are

$$
T=\frac{9 t}{\left(r^{3 / 2}+t^{3 / 2}\right)^{2}}, \quad 2 c m q=\frac{9 r}{\left(r^{3 / 2}+t^{3 / 2}\right)^{2}} .
$$

Figure 3 presents the time and the radial coordinate dependence of the temperature and the heat flux. These solutions are not discontinuous. Analytical and numerical calculus suggest that $\omega=-1 / 2$ is a critical exponent: for $-1<\omega \leq-1 / 2$ the solutions are continuous, for the shocks always appear $\omega>-1 / 2$.

\section{Summary}

We presented a hyperbolic model for heat conduction in solids where the relaxation time and heat conduction coefficients are power law functions of time. There are basically two different regimes available for different power laws. For $1<\omega \leq-1 / 2$ the solutions are continuous for all positive time and radial coordinate, for $\omega>-1 / 2$ the solutions are only continuous on a finite and closed [0: $\eta_{0]}$ interval and have a finite jump at the the endpoint $\eta_{0}$. As physical interpretation numerous

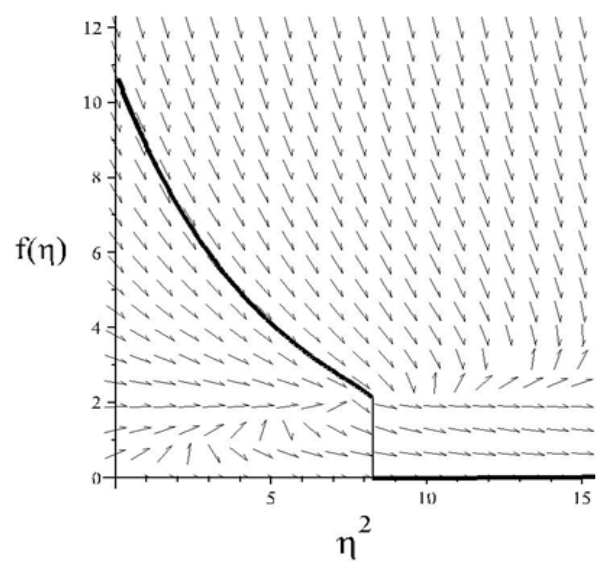

Figure 1: The direction field of (a) Eq. (17) for $!=0$ and (b) Eq. (16) for! $=0$ The solid line presents numerical solutions for a) $y(3)=0: 5$ and for b) $f(0)=10: 8$.

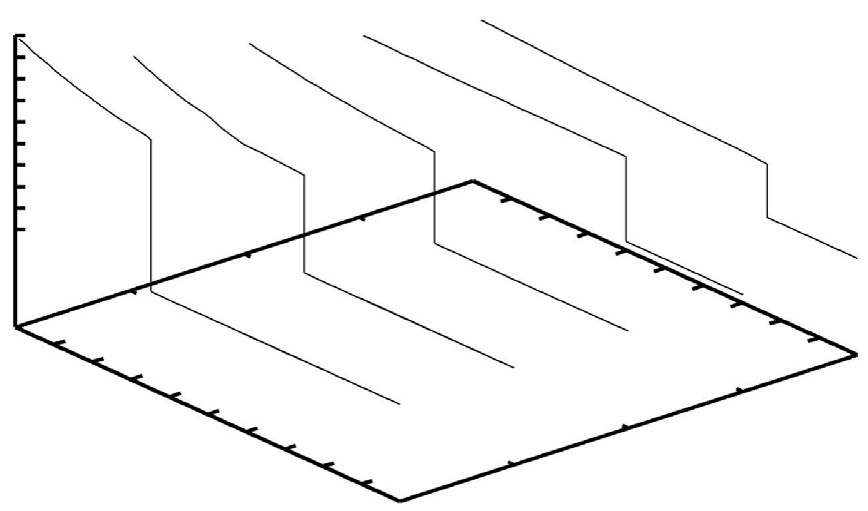

Figure 2: The shock-wave propagation of the temperature distribution of $T(r ; t)$ for $!=0$ 

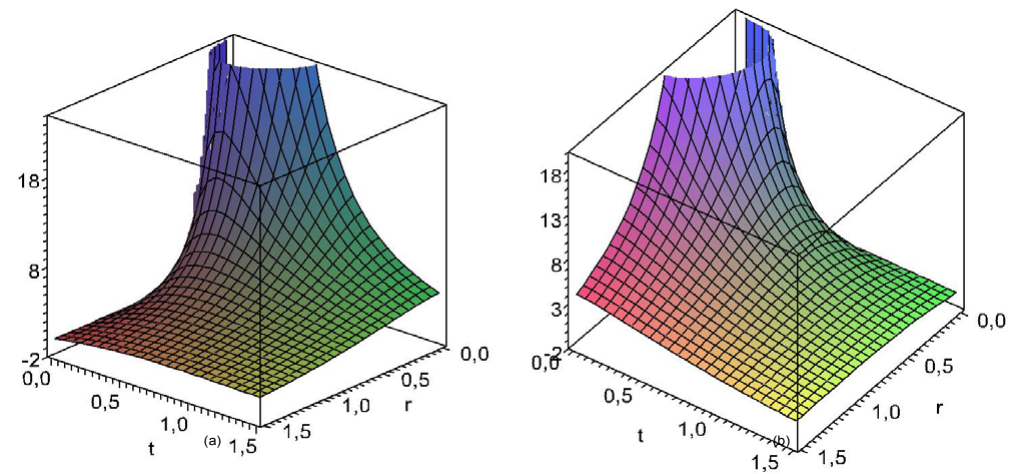

Figure 3: The continuous solutions: (a) the temperature distribution of Eq. (18) and (b) the heat flux distribution of Eq. (18)

materials and solid state systems were mentioned with temperature dependent heat conduction coefficients.

\section{References}

1. Maxwell JC (1867) On the Dynamical Theory of Gases. Trans R Soc Lond 157: 49-88.

2. Zel'dovich YB, Raizer YP (1965) Physics of Shock Waves and HighTemperature Hydrodynamic Phenomena (Academic New York).

3. Dubin DHE, O'Neil TM (1997) Cross-Magnetic-Field Heat Conduction in Nonneutral Plasmas Phys. Rev Lett 78: 3868.

4. Zeldovich YB, Kompaneets AS (1950) Collection Dedicated to the 70th Birthday of A.F. Joffe, Izdat. Akad. Nauk SSSR p: 61.

5. Chandrasekharaiah DS (1986) Appl. Mech. Rev 39: 355-376.

6. Cattaneo C (2001) Sulla conduzione del calore Atti. sem Mat. Fis. Univ. Modena.

7. Barna IF, Kersner R (2010) Heat conduction: a telegraph-type model with selfsimilar behavior of solutions. J. Phys. A Math. Theor 43: 375210.

8. Joseph DD, Preziosi L (1989) Heat waves Rev Mod Phys 61: 41.

9. Makai M (2011) On the speed of heat waves. Eur Phys Lett 96: 40010.

10. Jones H (1956) Handb. Phys 19: 227.
11. Ashcroft NW Mermin DN Solid State Physics Thomson Learning Inc.

12. Nettleton R E (1987) Non-linear heat conduction in dense fluids. Phys A: Math Gen 20: 4017.

13. Vadasz P (2006) Heat Conduction in Nanofluid Suspensions. J Heat Trans 128: $465-477$.

14. Small JP, Shi L, Kim P (2003) Mesoscopic thermal and thermoelectric measurements of individual carbon nanotubes. Solid. State. Commun 127: 181

15. Berber S, Kwon YK, Tománek D (2003) Unusually High Thermal Conductivity of Carbon Nanotubes. Phys. Rev. Lett 84: 4613.

16. Cahill DG, Ford WK, Goodson KE, Mahan GD, Mujumdar A, et al. (2003) Nanoscale thermal transport. Journ. Appl Phys. 93: 793.

17. Jang W, Chen Z, Bao W, Lau CN, Dames C (2010) Thickness-Dependent Thermal Conductivity of Encased Graphene and Ultrathin Graphite. Nano. Lett 10: 3909.

18. Balandin AA (2011) Thermal properties of graphene and nanostructured carbon materials. Nature Materials 10: 569-581.

19. Sedov L (1993) Similarity and Dimensional Methods in Mechanics, CRC Press.

20. Wilhelm HE, Choi SH (1975) Nonlinear hyperbolic theory of thermal waves in metals. J. Chem. Phys 63: 2119.
This article was originally published in a special issue, Recent Advances of Lie Theory in differential Geometry, in memory of John Nash handled by Editor. Dr. Princy Randriambololondrantomalala, Unversity of Antananarivo, Madagascar 